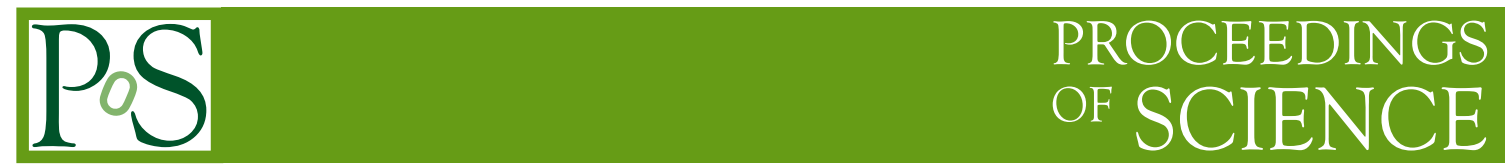

\title{
Phenomenology of light sterile neutrinos
}

\author{
Antonio Palazzo* \\ University of Bari and INFN \\ E-mail: palazzo@ba.infn.it
}

Several anomalies observed in short-baseline neutrino experiments suggest that the standard 3flavor framework may be incomplete and indicate the existence of light sterile neutrinos. Here, we present a brief review of the status of the neutrino oscillations within the $3+1$ scheme, the minimal extension of the standard 3-flavor framework with one sterile neutrino. We stress the potential role of LBL experiments in the searches of CP violation related to sterile neutrinos and their synergy with the SBL experiments.

XVII International Workshop on Neutrino Telescopes

13-17 March 2017

Venezia, Italy

${ }^{*}$ Speaker. 


\section{Introduction}

A long series of experiments performed in the last twenty years has established that neutrinos are massive and mix. Although the 3-flavor scheme has been identified as the only one able to accommodate simultaneously all data recorded in solar, atmospheric, reactor and accelerator neutrino experiments, a few anomalous results have been reported in short-baseline (SBL) neutrino oscillation measurements, which are not compatible with such a scheme (see [1] for a recent review). One possible interpretation of these anomalies involves new light sterile neutrinos (with mass in the $\mathrm{eV}$ range) which mix with the three ordinary active species.

In the simplest scenario, named $3+1$ scheme, only one sterile neutrino species is involved. The fourth mass eigenstate $v_{4}$ is separated from the standard "triplet" $\left(v_{1}, v_{2}, v_{3}\right)$ by a new large $\left(\sim 1 \mathrm{eV}^{2}\right)$ squared-mass, giving rise to the hierarchical spectrum $\left|\Delta m_{12}^{2}\right| \ll\left|\Delta m_{13}^{2}\right| \ll\left|\Delta m_{14}^{2}\right|$. The $4 \times 4$ mixing matrix can be parametrized as [2]

$$
U=\tilde{R}_{34} R_{24} \tilde{R}_{14} R_{23} \tilde{R}_{13} R_{12},
$$

where $R_{i j}\left(\tilde{R}_{i j}\right)$ is a real (complex) $4 \times 4$ rotation in the $(i, j)$ plane. The complex rotations depend on the CP-phases $\delta_{i j}$.

By construction, the $3+1$ scheme predicts sizable oscillation effects at the short baselines where the new frequency $\Delta_{14}=\Delta m_{14}^{2} L / 4 E$ ( $L$ being the baseline and $E$ the neutrino energy) is of order one. However, sterile neutrinos may leave their imprints also in non-short-baseline experiments. In these setups the new oscillations get averaged because they are very fast $\left(\Delta_{14} \gg 1\right)$ and the manifestation of the process of the active-sterile oscillations is more subtle.

In the solar sector, for example, the admixture of the electron neutrino with the $v_{4}$ mass eigenstate (parametrized by the matrix element $U_{e 4}$ ) leads to small deviations from the unitarity of the $\left(v_{1}, v_{2}, v_{3}\right)$ sub-system (see [3, 4, 5]). In the atmospheric neutrinos, as first evidenced in [6], at very high $[O(\mathrm{TeV})]$ energies, an MSW resonant behavior is expected, which leads to a modification of the zenith angle distributions. Finally, as first evidenced in [2], sterile neutrino oscillations can show up also in the long-baseline (LBL) accelerator experiments, where they produce to new interference phenomena. This circumstance is of particular interest in consideration of the world-wide program of new LBL facilities.

In what follows we briefly describe the anomalous SBL results and discuss the potential role of LBL experiments in the searches of CP violation (CPV) related to sterile neutrinos.

\section{The LSND and MiniBoone anomalies}

Accelerator experiments with baselines of a few tens of meters and neutrino energies of a few tens of $\mathrm{MeV}$ can effectively observe neutrino oscillations occurring at $\Delta m^{2} \sim 1 \mathrm{eV}^{2}$. Their results are usually interpreted in terms of a new mixing angle $\theta$ and a new mass-squared difference $\Delta m^{2}$. In the 3+1 framework the following identifications hold: $\Delta m^{2} \equiv \Delta m_{14}^{2}$ and $\sin ^{2} 2 \theta \equiv 4\left|U_{e 4}\right|^{2}\left|U_{\mu 4}\right|^{2}$. The anomalous result recorded at the LSND experiment [7] was the first datum pointing towards light sterile neutrinos. Such an experiment, designed to study $\bar{v}_{\mu} \rightarrow \bar{v}_{e}$ transitions, detected an excess of electron antineutrino events a the $\sim 3.8 \sigma$ level. The experiment KARMEN [8], having a design very similar to LSND, observed no such a signal, but was not able to rule out completely 
the mass-mixing parameter region allowed by LSND. The experiment MiniBooNE [9], sensitive both to $v_{\mu} \rightarrow v_{e}$ and $\bar{v}_{\mu} \rightarrow \bar{v}_{e}$ transitions, seems to lend support to the LSND finding.

An independent test of the LSND and MiniBooNE anomalies has been recently carried out at the long-baseline experiments ICARUS [10] and OPERA [11]. In these experiments, due to the high energy of the beam $(<E>\sim 17 \mathrm{GeV})$, the 3-flavor effects induced by non-zero $\theta_{13}$ are negligible. As a result, the experiments are sensitive to sterile neutrino oscillations, although these are completely averaged out due to the high value $L / E \sim 36.5 \mathrm{~m} / \mathrm{MeV}$, and appear as mere enhancement of the expected rate of events. Both collaborations have performed the analysis in an effective 2-flavor description. However, in [12] it was pointed out that in the $3+1$ scheme important corrections arise due to the presence of a new genuinely 4-flavor interference term in the transition probability. In addition, in the 4-flavor framework the $v_{e}$ beam background is not a fixed quantity like in the 2-flavor scheme. A correct 4-flavor analysis [12] of ICARUS and OPERA leads to a substantial weakening (by a factor of $\sim 3$ ) of the upper bounds on the sterile neutrino mixing. ICARUS and OPERA are not sensitive enough to rule out the mass-mixing region preferred by LSND and MiniBooNE, and can only restrict the allowed region to values of $\sin ^{2} 2 \theta<f e w \times 10^{-2}$.

\section{The reactor and gallium anomalies}

The re-calculations of the reactor antineutrino spectra performed in [13, 14] have given new impetus to the study of light sterile neutrinos. These calculations point towards fluxes which are $\sim$ $3.5 \%$ higher than previous estimates and have raised the so-called reactor antineutrino anomaly [15]. In fact, adopting these new fluxes, the SBL reactor measurements show a deficit with respect to the theoretical expectations.

The recent high statistics measurements of the antineutrino spectra performed by Daya Bay [16], Double Chooz [17] and RENO [18] (and more recently also by NEOS [19]) have evidenced an unexpected bump around $5 \mathrm{MeV}$ in the prompt energy spectrum, deviating from the predictions at the $\sim 4 \sigma$ level. The bump structure appears to be similar at the near and far detectors and is positively correlated with the reactor power. This strongly disfavors a possible explanation in terms of new-physics (for example super-light sterile neutrinos [20]). This unexpected feature of the spectrum evidences that our understanding of the reactor spectra is incomplete and reinforces the case for a revision of the reactor flux predictions and their error budget. To this regard, it has been recently pointed out [21] (see also [22]), that if one hypothesizes that the reactor anomaly is not due to active-sterile neutrino oscillations, it can be explained entirely by a miscalculation of the ${ }^{235} \mathrm{U}$ reactor antineutrino flux.

An apparently unrelated deficit has been observed in the solar neutrino experiments GALLEX and SAGE [23] using high intensity radioactive sources. The statistical significance of the deficit fluctuates around the $3 \sigma$ level slightly depending on the assumptions made on the theoretical estimate of the cross section $v_{e}+{ }^{71} \mathrm{Ga} \rightarrow{ }^{71} \mathrm{Ge}+e^{-}$. While the anomaly is compatible with a signal of new physics, both a systematic error in the Ge extraction efficiency or in the theoretical estimate of the cross-section remain credible alternative explanations.

Both the reactor and gallium anomalies can be interpreted in terms of a phenomenon of electron neutrino disappearance driven by sterile neutrino oscillations. In an effective 2-flavor scheme the results can be described by a new mass-squared difference $\Delta m^{2}$ and an effective mixing angle 
$\theta$. In a $3+1$ framework the following identifications hold: $\Delta m^{2} \equiv \Delta m_{14}^{2}$ and $\sin ^{2} 2 \theta \equiv 4\left|U_{e 4}\right|^{2}(1-$ $\left.\left|U_{e 4}\right|^{2}\right)$. The simultaneous explanation of both anomalies requires values of $\Delta m^{2} \sim 1.7 \mathrm{eV}^{2}$ and $\sin ^{2} 2 \theta \sim 0.1$ (see [24]). The inclusion of the recent results from the reactor experiment NEOS [19] tends to shift downward the best fit value of the mixing angle to $\sin ^{2} 2 \theta \sim 0.08$ (see [24]). It should be noted that, if the best fit point lies in the region indicated by NEOS, the detection of a signal at the future SBL experiments will be more challenging than originally envisaged.

The results of NEOS deserve some further comment. A raster scan analysis made by the collaboration excludes the hypothesis of oscillations at the $90 \%$ C.L. On the other hand, the expansion of the $\Delta \chi^{2}$ evidences a preference for sterile neutrino oscillations at roughly $2 \sigma$ in 2 d.o.f. (standard 3-flavor case disfavored at $\Delta \chi^{2}=6.5$ ). In addition, the region of parameters identified by NEOS lies inside the region allowed by all the current SBL data (see [24]). We think that these two findings are intriguing and deserve more attention.

\section{Sterile neutrinos at long-baseline experiments}

The short-baseline experiments are undoubtedly the best place where to seek for sterile neutrinos and certainly, if a breakthrough will come, it will take place at a SBL experiment. However, the SBL experiments have an intrinsic limitation, which would hamper the further study of the properties of the $3+1$ scheme. In particular, they are insensitive to the three CP-violation phases involved in such a scheme. In fact, CP-violation is a genuine 3-flavor phenomenon, whose observation requires the sensitivity to the interference between at least two independent oscillation frequencies. In a SBL experiment only the new largest oscillation frequency $\left(\Delta_{14} \sim 1\right)$ is visible, while both the atmospheric and the solar splittings are basically unobservable $\left(\Delta_{13} \simeq \Delta_{12} \simeq 0\right)$. Therefore, this class of experiments is blind to CP-violation effects. ${ }^{1}$ Therefore, other kinds of experiments are necessary to access the $\mathrm{CP}$ violation induced by sterile neutrinos. We are lucky because these experiments already exist. We are referring to the LBL experiments, both those already operational and the planned ones. As a matter of fact, although such experiments were originally designed to seek the standard CP-phase $\delta$, they are also capable to provide information about other sources of $\mathrm{CP}$ violation. This is not obvious a priori and it is true only because, as we will see below, a new interference term arises in the presence of sterile neutrinos, which has exactly the same order of magnitude of the standard 3-flavor interference term.

We recall that the LBL experiments, when working in the $v_{\mu} \rightarrow v_{e}$ (and $\bar{v}_{\mu} \rightarrow \bar{v}_{e}$ ) appearance channel are sensitive to the 3-flavor CPV because, at long baselines, the $v_{\mu} \rightarrow v_{e}$ transition amplitude develops an interference term between the atmospheric $\left(\Delta m_{13}^{2}\right.$-driven) and the solar $\left(\Delta m_{12^{-}}^{2}\right.$ driven) oscillations, which depends on the CP-phase $\delta$. As first evidenced in [2], in the presence of sterile neutrinos a new interference term arises, which depends not only from $\delta \equiv \delta_{13}$ but also from one new CP-phase ( $\left.\delta_{14}\right)$. From the discussion made in [2] (see also [25]), it emerges that the transition probability can be approximated as the sum of three terms

$$
P_{\mu e}^{4 v} \simeq P^{\mathrm{ATM}}+P_{\mathrm{I}}^{\mathrm{INT}}+P_{\mathrm{II}}^{\mathrm{INT}} .
$$

\footnotetext{
${ }^{1}$ In the $3+N_{s}$ schemes with $N_{s}>1, \mathrm{CPV}$ could be observed at SBL experiments. However, these setups can probe only a limited number of all the $\mathrm{CP}$ phases involved in the model. In contrast, LBL experiments have access to all such phases. For example, in the $3+2$ scheme, the SBL experiments are sensitive only to one CP-phase over a total of five observable CP-phases.
} 
The first term represents the positive-definite atmospheric transition probability, while the other two terms (which can assume both positive and negative values) are due to interference. The first of them is related to the well-known standard atmospheric-solar interference, while the second is driven by the atmospheric-sterile interference. We now observe that the transition probability depends on the three small mixing angles $\theta_{13}, \theta_{14}, \theta_{24}$, and that it occurs that these mixing angles have the same order of magnitude $\varepsilon \sim 0.15$. In addition, we note that the ratio of the solar and atmospheric squared-mass splittings $\alpha \equiv \Delta m_{12}^{2} / \Delta m_{13}^{2} \simeq \pm 0.03$, which is of order $\varepsilon^{2}$. Keeping terms up to the third order, in vacuum, one finds [2]

$$
\begin{aligned}
& P^{\mathrm{ATM}} \simeq 4 s_{23}^{2} s_{13}^{2} \sin ^{2} \Delta, \\
& P_{\mathrm{I}}^{\mathrm{INT}} \simeq 8 s_{13} s_{12} c_{12} s_{23} c_{23}(\alpha \Delta) \sin \Delta \cos \left(\Delta+\delta_{13}\right), \\
& P_{\mathrm{II}}^{\mathrm{INT}} \simeq 4 s_{14} s_{24} s_{13} s_{23} \sin \Delta \sin \left(\Delta+\delta_{13}-\delta_{14}\right),
\end{aligned}
$$

where $s_{i j} \equiv \sin \theta_{i j}, c_{i j} \equiv \cos \theta_{i j}$ and $\Delta \equiv \Delta m_{13}^{2} L / 4 E$ is the atmospheric oscillating frequency. The matter effects slightly modify the transition probability leaving the picture described above almost unaltered (see $[2,25]$ for details).

Remarkably, for typical values of the mixing angles preferred by the current global $3+1$ fits [24], the amplitude of the new interference term is almost identical to that of the standard one. As a consequence, one expects some sensitivity of the LBL experiments NOvA [26] and $\mathrm{T} 2 \mathrm{~K}$ [27] to the non-standard CP-phase $\delta_{14}$. Therefore, these two experiments and their constraints on the CP-phase $\delta_{14}$, should be included in any accurate analysis of the $3+1$ scheme. This has been done in the work [28], where a joint analysis of SBL and LBL data has been presented for the first time.

Figure 1, taken from such a work, displays the projections of the $\Delta \chi^{2}$ for inverted hierarchy (IH). The left-bottom panel reports the projection on the plane of the two mixing angles $\left(\theta_{14}, \theta_{24}\right)$. The other two panels display the constraints in the plane formed by each one of the two mixing angles and the new CP-phase $\delta_{14}$. Similar results (not shown) are obtained for normal hierarchy (NH). The three contours are drawn for $\Delta \chi^{2}=2.3,4.6,6.0$, corresponding to $68 \%, 90 \%$ and $95 \%$ C.L. for 2 d.o.f. The overall goodness of fit is acceptable ( $\mathrm{GoF}=24 \%$ ), while the parameter goodness of fit, which measures the statistical compatibility between the appearance and disappearance data sets, is lower $(\mathrm{GoF}=7 \%)$. This implies that even if the closed contours presented for the two new mixing angles $\theta_{14}$ and $\theta_{24}$ exclude the 3-flavor case with high significance (slightly more than six standard deviations), one cannot naively interpret this circumstance as an evidence for sterile neutrinos. In addition, we recall that light sterile neutrinos, unless endowed with new properties, are in tension with cosmological data.

Given that NOvA and T2K posses already a weak sensitivity to the new CP-phase $\delta_{14}$, it is very interesting to ask how things will improve at the planned LBL experiments. This issue has been investigated in detail in the works [29, 30, 31] (see also [32, 33]). In Fig. 2, taken from [30], we provide an illustrative example concerning the DUNE experiment. The bands displayed in the left, middle and right panels represent the discovery potential of the CPV induced, respectively, by $\delta_{13}, \delta_{14}$ and $\delta_{34}$. The thinner (magenta) bands correspond to the case in which all the three new mixing angles have the same value $\theta_{14}=\theta_{24}=\theta_{34}=9^{0}$. The thicker (green) bands correspond to the situation in which $\theta_{14}=\theta_{24}=9^{0}$ and $\theta_{34}=30^{\circ}$. In each panel, the bands were obtained 


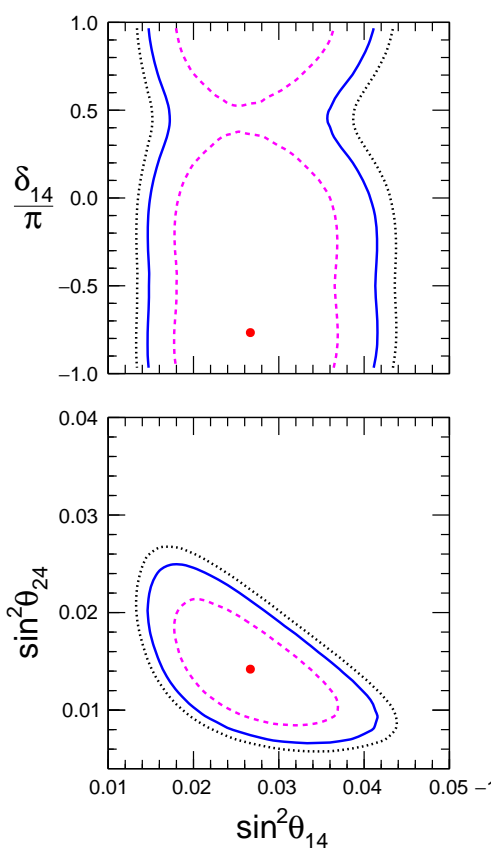

\section{Inverted hierarchy}

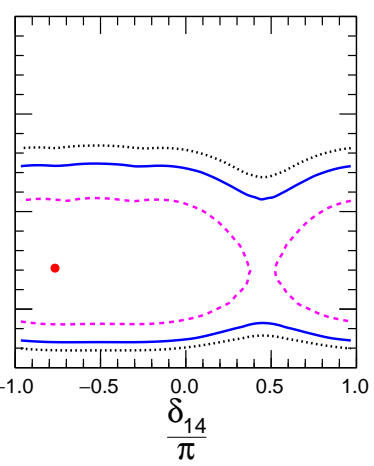

Figure 1: Regions allowed by the combination of the SBL and LBL data (NOvA and T2K) together with the $\theta_{13}$-sensitive reactor results. Figure taken from [28].

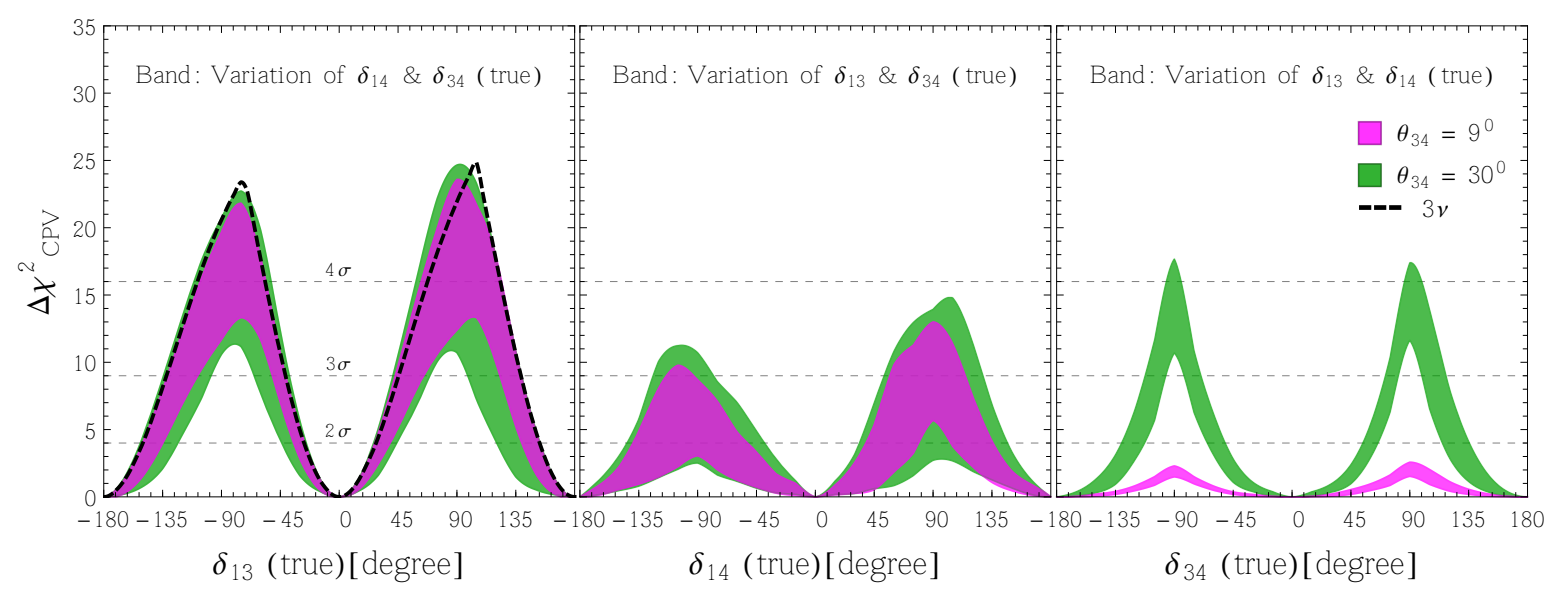

Figure 2: DUNE discovery potential of the CPV induced by the three CP phases involved in the $3+1$ scheme. See the text for details. Figure taken from [30].

by varying the true values of the two undisplayed $\mathrm{CP}$-phases in the range $[-\pi, \pi]$. In all cases, marginalization over the hierarchy was performed with $\mathrm{NH}$ as true choice. From Fig. 2 we learn that the sensitivity to $\delta_{14}$ will substantially increase at DUNE at the price of loosing some information on the standard CP phase $\delta_{13}$. 


\section{Conclusions}

We have presented a brief discussion of the current phenomenology of light sterile neutrinos. In the eventuality of a discovery at a new short-baseline experiment we will face the challenge of determining all the parameters that govern the extended framework and in particular its new CPviolating phases. In this arena LBL experiments can give an important contribution being sensitive to new CP-violation phenomena. Therefore, the two classes of experiments (SBL and LBL) will be synergic in the searches of sterile neutrinos.

\section{Acknowledgments}

I would like to thank the organizers for the very stimulating and enjoyable conference. This work is supported by the project Beyond three neutrino families within the FutureInResearch program, Fondo di Sviluppo e Coesione 2007-2013, APQ Ricerca Regione Puglia "Programma regionale a sostegno della specializzazione intelligente e della sostenibilità sociale ed ambientale". I also acknowledge support by the research project TAsP funded by the Instituto Nazionale di Fisica Nucleare (INFN).

\section{References}

[1] A. Palazzo, Mod. Phys. Lett. A 28, 1330004 (2013).

[2] N. Klop and A. Palazzo, Phys. Rev. D 91 no. 7, 073017 (2015).

[3] A. Palazzo, Phys. Rev. D 83, 113013 (2011).

[4] A. Palazzo, Phys. Rev. D 85, 077301 (2012).

[5] C. Giunti and Y. F. Li, Phys. Rev. D 80, 113007 (2009).

[6] H. Nunokawa, O. L. G. Peres and R. Zukanovich Funchal, Phys. Lett. B 562, 279 (2003).

[7] C. Athanassopoulos et al. [LSND Collaboration], Phys. Rev. Lett. 773082 (1996).

[8] B. Armbruster et al. [KARMEN Collaboration], Phys. Rev. D 65112001 (2002).

[9] A. A. Aguilar-Arevalo et al., arXiv:1207.4809 [hep-ex].

[10] M. Antonello et al. Eur. Phys. J. C 73 no. 3, 2345 (2013).

[11] N. Agafonova et al. [OPERA Collaboration], JHEP 1307, 004 (2013) [Addendum-ibid. 1307 085].

[12] A. Palazzo, Phys. Rev. D 91 no. 9, 091301 (2015).

[13] T. A. Mueller et al., Phys. Rev. C 83054615 (2011).

[14] P. Huber, Phys. Rev. C 84024617 (2011) [2012 Erratum-ibid. 85029901 (2011)].

[15] G. Mention et al. Phys. Rev. D 83073006 (2011).

[16] F. P. An et al. [Daya Bay Collaboration], Chin. Phys. C 41, no. 1, 013002 (2017).

[17] S. Schoppmann [Double Chooz Collaboration], PoS HQL 2016, 010 (2017).

[18] S. H. Seo et al. [RENO Collaboration], arXiv:1610.04326 [hep-ex].

[19] Y. J. Ko et al., Phys. Rev. Lett. 118, no. 12, 121802 (2017). 
[20] A. Palazzo, JHEP 1310, 172 (2013).

[21] C. Giunti, Phys. Lett. B 764, 145 (2017).

[22] F. P. An et al. [Daya Bay Collaboration], [arXiv:1704.01082 [hep-ex]].

[23] J. N. Abdurashitov et al., Phys. Rev. C, 73 (2006) 045805.

[24] S. Gariazzo, C. Giunti, M. Laveder and Y. F. Li, arXiv:1703.00860 [hep-ph].

[25] A. Palazzo, Phys. Lett. B, 757142 (2016).

[26] P. Adamson et al. [NOvA Collaboration], arXiv:1703.03328 [hep-ex].

[27] K. Abe et al. [T2K Collaboration], Phys. Rev. Lett. 118, no. 15, 151801 (2017).

[28] F. Capozzi, C. Giunti, M. Laveder and A. Palazzo, Phys. Rev. D 95, no. 3, 033006 (2017).

[29] S. K. Agarwalla, S. S. Chatterjee, A. Dasgupta and A. Palazzo, JHEP 1602, 111 (2016).

[30] S. K. Agarwalla, S. S. Chatterjee and A. Palazzo, JHEP 1609, 016 (2016).

[31] S. K. Agarwalla, S. S. Chatterjee and A. Palazzo, Phys. Rev. Lett. 118, no. 3, 031804 (2017).

[32] J. M. Berryman, A. de Gouvea, K. J. Kelly and A. Kobach, Phys. Rev. D 92, no. 7, 073012 (2015).

[33] D. Dutta, R. Gandhi, B. Kayser, M. Masud and S. Prakash, JHEP 1611, 122 (2016). 